\title{
Surgical repair of traumatic medial disruption of the elbow in competitive athletes
}

\author{
Goro Inoue, MD and Yoshio Kuwahata MD \\ Department of Orthopaedic Surgery, Nagoya University Branch Hospital, Nagoya 461 and ${ }^{\dagger}$ Kuwahata \\ Orthopaedic Clinic, Shizuoka 437-16, Japan
}

\begin{abstract}
Five active athletes with acute medial elbow rupture were treated with muscle-ligamentous repair and a spiked washer. All patients regained full strength as well as stability of the elbow, and resumed previous sporting activities within 3 months of surgery. Early surgical repair of the ligament and flexor mass should be considered for active athletes who exhibit gross instability of the elbow on a valgus stress test without anaesthesia.
\end{abstract}

Keywords: medial collateral ligament, flexor muscles, instability, spiked washer

Most authors agree that acute isolated rupture of the medial collateral ligament of the elbow, with or without dislocation of the elbow, is well treated by a short period of immobilization ${ }^{1-4}$. However, when there is proven gross instability of the elbow following extensive rupture of the medial soft tissues, the elbow must be immobilized for 3 weeks or more and protected against valgus stress until ligamentous healing is complete. This may lead to stiffness and weakness of the elbow. Full recovery of motion and strength takes more than 6 months in most patients ${ }^{1}$, and is likely to cause prolonged disability. Some patients may be willing and able to endure a long period of disability. However, active or professional athletes who are eager to return to competitive sports, are given the option of earlier surgical repair to shorten the period of disability.

This paper reports on five active athletes with acute medial elbow ruptures who were treated by surgical repair of the ligament and flexors.

\section{Materials and methods}

Four of the patients were men and one a woman; ranging in age from 17-29. The four men injured their elbows by a fall onto the extremity during a motorcross race. The woman received valgus stress to the elbow by a direct blow while playing volleyball. Three of the injuries were associated with lateral dislocation of the elbow, which was reduced by help from a friend, and two had no associated injuries.

Address for correspondence: Dr Goro Inoue, Department of Orthopaedic Surgery, Nagoya University Branch Hospital, 1-1-20 Daikominami, Higashi-ku, Nagoya 461, Japan
Three cases involved the left elbow and two the right elbow. All were seen at our clinic within $24 \mathrm{~h}$ of injury. Clinical examination of each patient showed generalized swelling with ecchymosis on the medial side of the elbow and gross medial instability without anaesthesia (Figure 1.) Signs of ulnar nerve irritation were observed in three patients. Plain radiographs showed a small avulsion fracture of the medial epicondyle in one patient. Unanaesthetized valgus stress radiographs showed significant medial instability in all patients (Figure 2).

The operation was done within 3 days of injury in all patients. Through a medial approach, the ulnar nerve was identified and preserved. In each patient, the flexor muscles and ulnar collateral ligament were torn from the medial epicondyle, and the joint capsule was also torn at the antero-medial side of the elbow (Figure 3). The medial elbow joint was inspected, and debridement of the joint was carried out. The torn capsule was repaired by interrupted sutures. The torn flexor muscle origin and ulnar collateral ligament were reattached to the medial

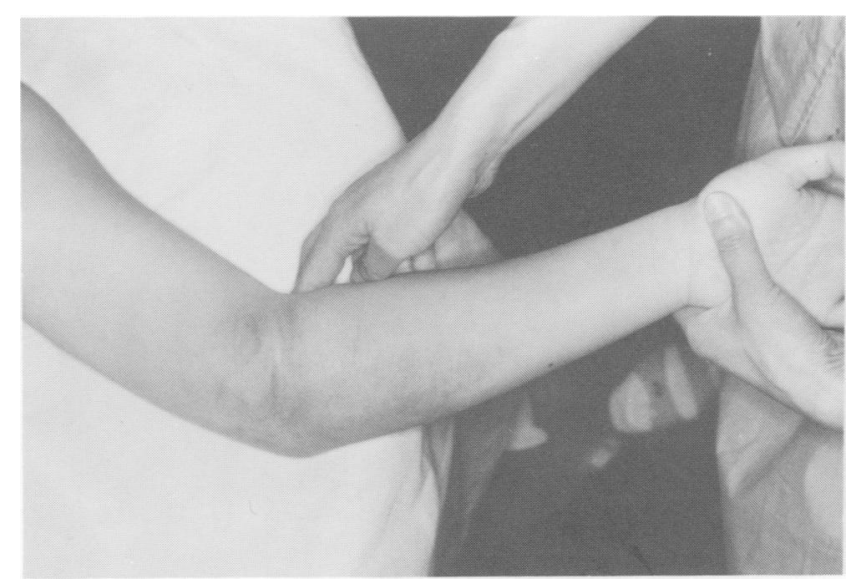

Figure 1. The elbow demonstrates gross instability on a valgus stress without anaesthesia 


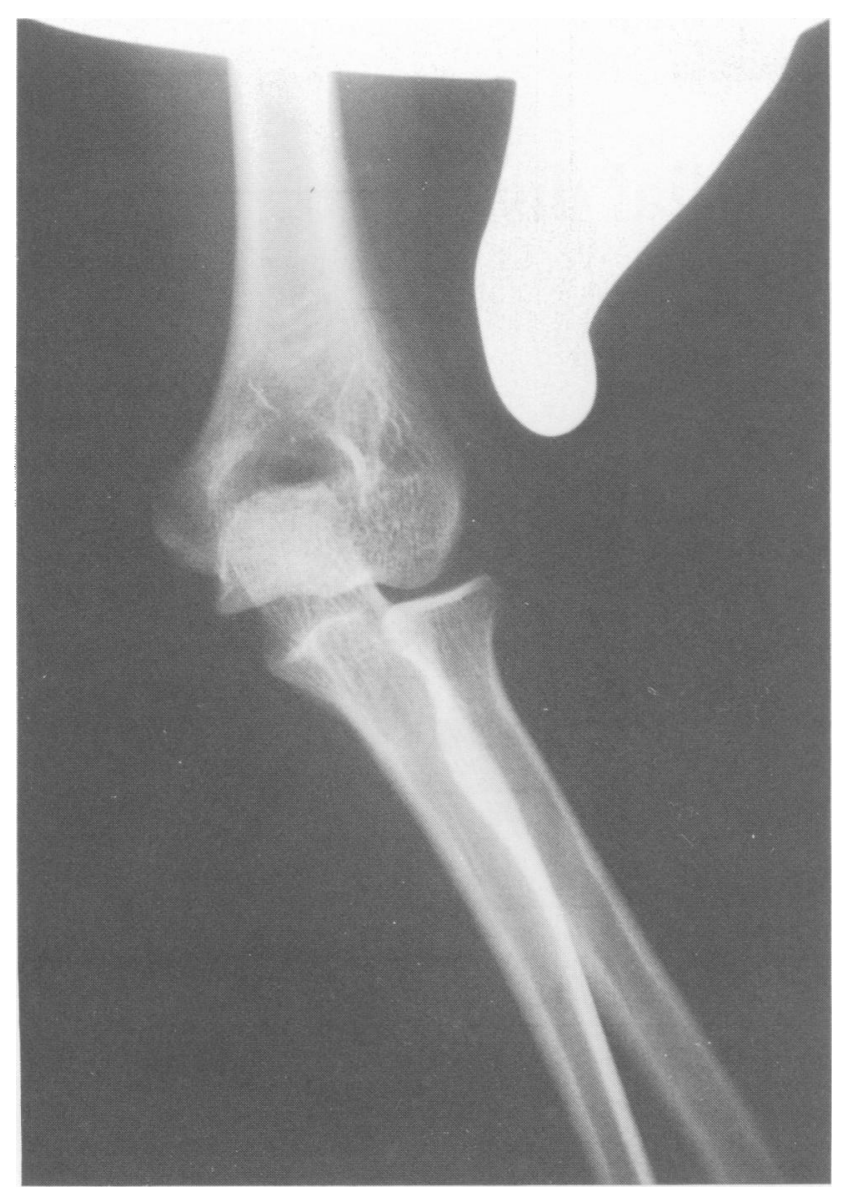

Figure 2. Unanaesthetized valgus stress radiograph of an individual with medial elbow rupture showing a wide gap at the medial aspect of the ulnohumeral joint epicondyle using a spiked washer and screw. The ulnar nerve was decompressed in three patients (Figure 4). A Penrose drain was placed in the joint, which was removed 3 days postoperatively. The elbows were placed in a long posterior splint for one week, after which the patients started unprotected movement of the elbow joint. All patients regained full strength as well as stability of the elbow, and returned to their previous sports activities within 3 months of surgery (Figure 5). The minimum followup period was one year, with an average of 16 months. All patients had normal range of motion of the elbow with no pain and did well at their previous competitive sports. Radiographs revealed no myositis ossificans or degenerative joint changes (Figure 6). No symptoms of residual ulnar nerve dysfunction could be detected.

\section{Discussion}

Management of elbow instability is controversial. Lasinger et al. ${ }^{3}$ and Josefsson et al. ${ }^{4}$, based on studies of surgical treatment and non-surgical treatment groups, recommended non-surgical treatment with immobilization in a cast for 2-3 weeks, while others ${ }^{5-7}$ recommended primary surgical repair of the ligament and early motion of the elbow. Mehlhoff $e t$ al. ${ }^{8}$ reported that after conservative treatment of simple dislocation of the elbow, symptoms of mild instability were seen in approximately $35 \%$ of patients. The symptoms, however, were not so severe as to prevent daily activity. For athletes, early return to competition with normal function of the elbow is the most important concern. Residual ligamentous instability may render this impossible.

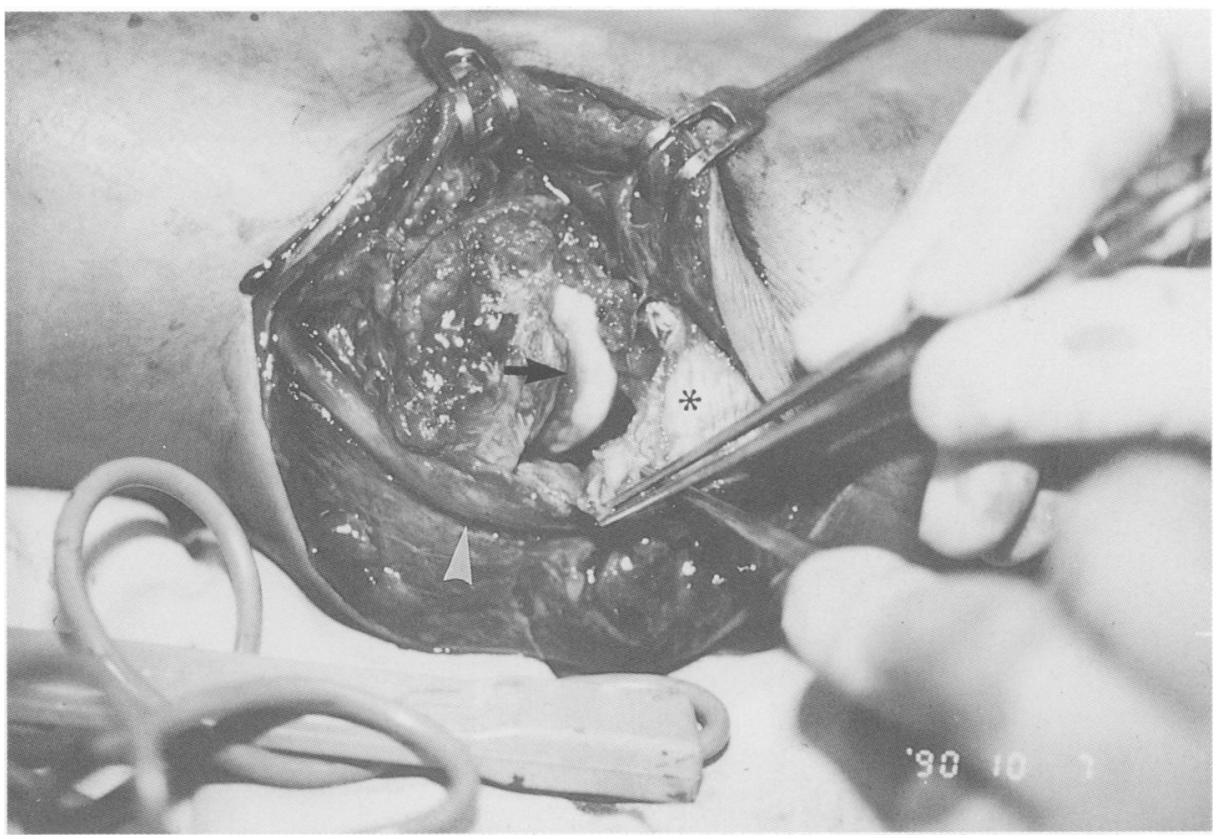

Figure 3. Complete rupture of the medial soft tissues: medial condyle (black arrow), ulnar nerve (white arrow), ruptured flexor mass (asterisks), instruments holding ruptured medial collateral ligament, torn at proximal attachment 
Figure 4. Muscle-ligamentous structures have been reattached to the medial epicondyle using a spiked washer and screw (black arrow). The ulnar nerve has been decompressed by the dissection of the cubital tunnel and fasciotomy of the flexor carpi ulmaris (white arrows)
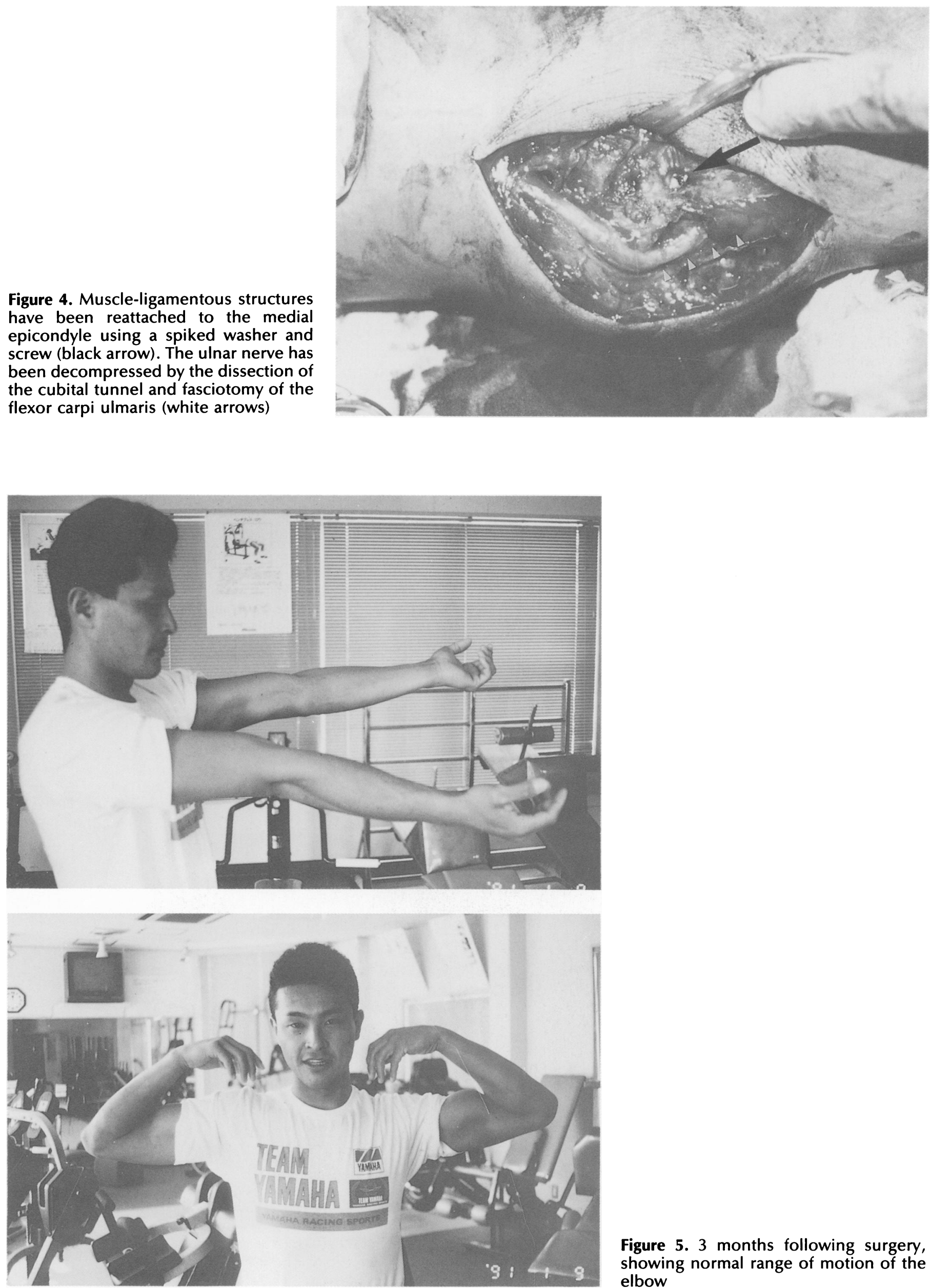

Figure 5. 3 months following surgery, showing normal range of motion of the elbow 


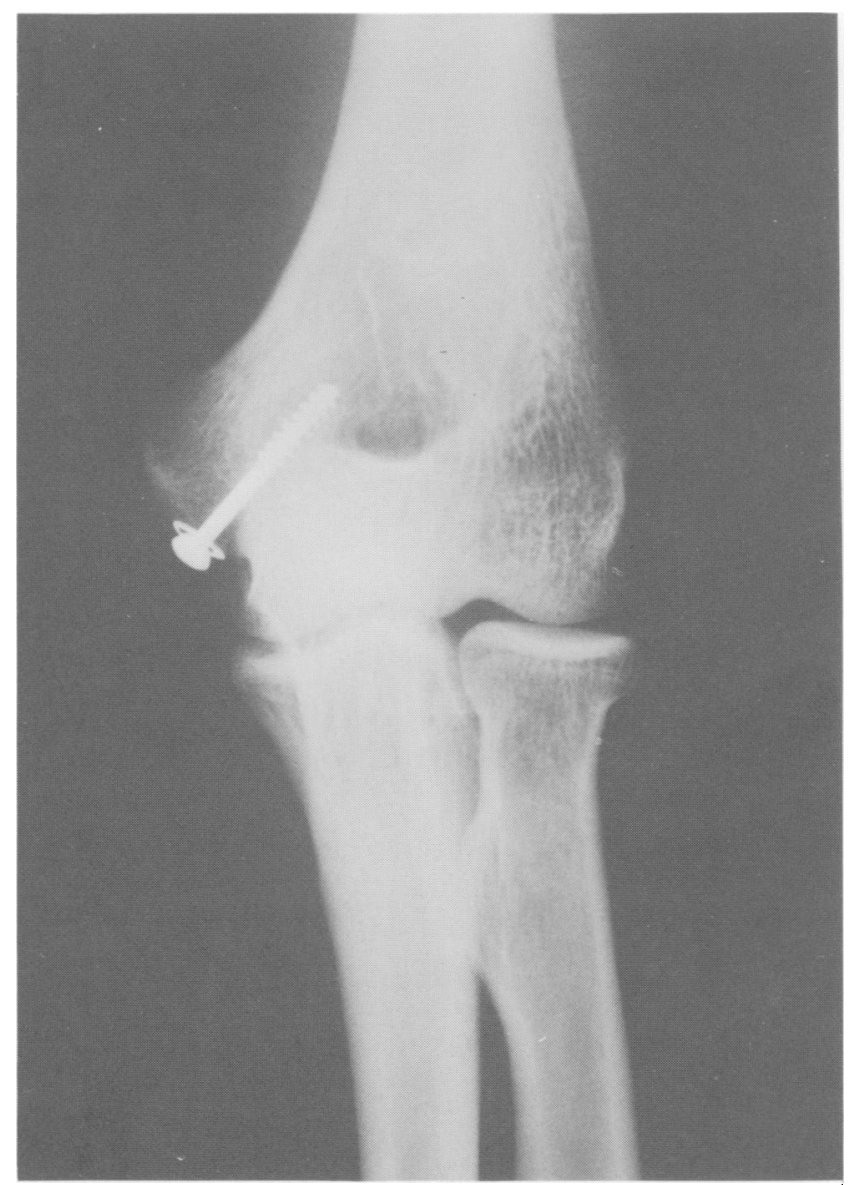

Figure 6. Radiograph at 1 year after surgery showing no evidence of myositis ossificans or degenerative changes

Stability of the elbow joint varies according to the degree of injury to the medial collateral ligaments, capsule and flexor mass. Isolated rupture of the medial collateral ligament is usually stable when the patient is examined without anaesthesia due to resistance of the intact flexor muscle mass. When this injury is associated with extensive rupture of the flexor muscle, the elbow demonstrates gross instability to valgus stress even without anaesthesia. The authors thus consider muscle-ligament repair to be indicated when unanaesthetized patients exhibit gross instability of the elbow on a valgus stress test.

There are many suture techniques for repair of torn ligaments. Torn ligaments and the flexor mass at the elbow are usually reinserted by direct suture, transosseous suture or a small staple. We prefer a spiked washer since it provides firmer attachment compared to other techniques.

Early surgical repair of ligaments and the flexor mass provides many advantages: (1) firm muscleligamentous repair using a spiked washer and screw can allow early motion of the elbow and early return to vigorous sports activities; (2) drainage for intraarticular haematoma can reduce pain in the joint and the potential for intra-articular adhesions; (3) the articular surface of the elbow and ulnar nerve, often involved in this injury, can be directly visualized and adequately managed if damaged.

\section{References}

1 Linscheid RL, Wheeler DK. Elbow dislocations. JAMA 1965; 194: 1171-6.

2 Wadsworth TJ. The Elbow. Edinburgh, UK: Churchill Livingstone 1982: 216-19.

3 Lansinger O, Karlsson J, Korner L, Mare K. Dislocation of the elbow joint. Arch Orthop Trauma Surg 1984; 102: 183-6.

4 Josefsson PO, Gentz C-F, Johnell O, Wendeberg B. Surgical versus non-surgical treatment of ligamentous injuries following dislocation of the the elbow joint. J Bone Joint Surg 1987; 69(A): 605-8.

5 Marshall DJ. A review of anatomy, mechanism and sequelae of elbow dislocation. J Bone Joint Surg 1976; 58(B): 257-8.

6 Dürig M, Müller W, Rüedi TP, Gauer EF. The operative treatment of elbow dislocation in the adult. J Bone Joint Surg 1979; 61(A): 239-44.

7 Norwood LA, Shook JA, Andrews JR. Acute medial elbow ruptures. Am J Sports Med 1981; 9: 16-19.

8 Mehlhoff TL, Noble PC, Bennett JB, Tullos HS. Simple dislocation of the elbow in the adult. J Bone Joint Surg 1988; 70(A): 244-9. 